\title{
INDICATIONS OF INTERMEDIATE-SCALE ANISOTROPY OF COSMIC RAYS WITH ENERGY GREATER THAN 57 EeV IN THE NORTHERN SKY MEASURED WITH THE SURFACE DETECTOR OF THE TELESCOPE ARRAY EXPERIMENT
}

R. U. Abbasi ${ }^{1}$, M. Abe ${ }^{2}$, T. Abu-Zayyad ${ }^{1}$, M. Allen ${ }^{1}$, R. Anderson ${ }^{1}$, R. Azuma ${ }^{3}$, E. Barcikowski ${ }^{1}$, J. W. Belz ${ }^{1}$,

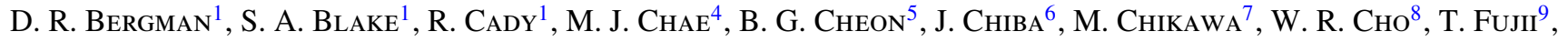

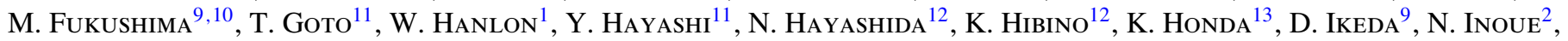
T. IshiI ${ }^{13}$, R. Ishimori ${ }^{3}$, H. Ito ${ }^{14}$, D. Ivanov ${ }^{1}$, C. C. H. Jui ${ }^{1}$, K. Kadota ${ }^{15}$, F. Kakimoto ${ }^{3}$, O. Kalashev ${ }^{16}$, K. Kasahara $^{17}$, H. KaWAi ${ }^{18}$, S. KaWAKami ${ }^{11}$, S. KaWAna ${ }^{2}$, K. KaWATA ${ }^{9}$, E. KIDo ${ }^{9}$, H. B. Kim ${ }^{5}$, J. H. Kim ${ }^{1}$, J. H. Kim ${ }^{19}$, S. Kitamura ${ }^{3}$,

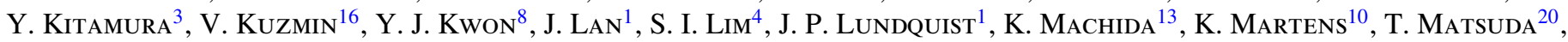
T. Matsuyama ${ }^{11}$, J. N. Matthews ${ }^{1}$, M. Minamino ${ }^{11}$, K. Mukai ${ }^{13}$, I. Myers $^{1}$, K. Nagasawa ${ }^{2}$, S. Nagataki ${ }^{14}$, T. Nakamura ${ }^{21}$,

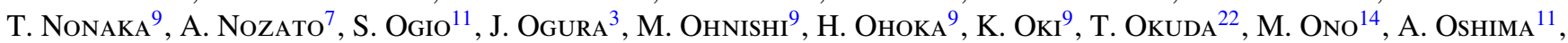
S. OzaWA ${ }^{17}$, I. H. Park ${ }^{23}$, M. S. Pshirkov ${ }^{24}$, D. C. Rodriguez ${ }^{1}$, G. RubTsov ${ }^{16}$, D. Ryu ${ }^{19}$, H. Sagawa ${ }^{9}$, N. Sakurai ${ }^{11}$, A. L. Sampson ${ }^{1}$, L. M. ScotT ${ }^{25}$, P. D. Shah ${ }^{1}$, F. Shibata ${ }^{13}$, T. Shibata ${ }^{9}$, H. Shimodaira ${ }^{9}$, B. K. Shin ${ }^{5}$, J. D. Smith ${ }^{1}$, P. Sokolsky ${ }^{1}$, R. W. Springer ${ }^{1}$, B. T. Stokes ${ }^{1}$, S. R. Stratton ${ }^{1,25}$, T. A. Stroman ${ }^{1}$, T. SuZawa ${ }^{2}$, M. Takamura ${ }^{6}$,

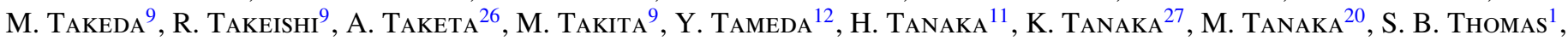
G. B. Thomson ${ }^{1}$, P. Tinyakov ${ }^{16,24}$, I. TKachev ${ }^{16}$, H. ToKuno ${ }^{3}$, T. Tomida ${ }^{28}$, S. Troitsky ${ }^{16}$, Y. Tsunesada $^{3}$, K. Tsutsumi $^{3}$,

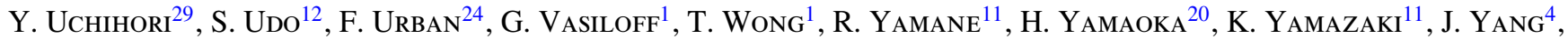

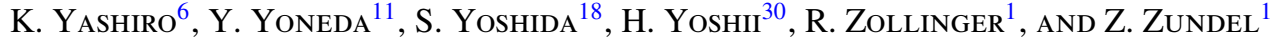

${ }^{1}$ High Energy Astrophysics Institute and Department of Physics and Astronomy, University of Utah, Salt Lake City, UT, USA

${ }^{2}$ The Graduate School of Science and Engineering, Saitama University, Saitama, Saitama, Japan

${ }^{3}$ Graduate School of Science and Engineering, Tokyo Institute of Technology, Meguro, Tokyo, Japan

${ }^{4}$ Department of Physics and Institute for the Early Universe, Ewha Womans University, Seodaaemun-gu, Seoul, Korea

${ }_{5}^{5}$ Department of Physics and The Research Institute of Natural Science, Hanyang University, Seongdong-gu, Seoul, Korea ${ }^{6}$ Department of Physics, Tokyo University of Science, Noda, Chiba, Japan ${ }^{7}$ Department of Physics, Kinki University, Higashi Osaka, Osaka, Japan

${ }^{8}$ Department of Physics, Yonsei University, Seodaemun-gu, Seoul, Korea

${ }^{9}$ Institute for Cosmic Ray Research, University of Tokyo, Kashiwa, Chiba, Japan

${ }^{10}$ Kavli Institute for the Physics and Mathematics of the Universe (WPI), Todai Institutes for Advanced Study, the University of Tokyo, Kashiwa, Chiba, Japan

${ }^{11}$ Graduate School of Science, Osaka City University, Osaka, Osaka, Japan

${ }^{12}$ Faculty of Engineering, Kanagawa University, Yokohama, Kanagawa, Japan

${ }^{13}$ Interdisciplinary Graduate School of Medicine and Engineering, University of Yamanashi, Kofu, Yamanashi, Japan

${ }^{14}$ Astrophysical Big Bang Laboratory, RIKEN, Wako, Saitama, Japan

${ }^{15}$ Department of Physics, Tokyo City University, Setagaya-ku, Tokyo, Japan

${ }^{16}$ Institute for Nuclear Research of the Russian Academy of Sciences, Moscow, Russia

${ }^{17}$ Advanced Research Institute for Science and Engineering, Waseda University, Shinjuku-ku, Tokyo, Japan

${ }^{18}$ Department of Physics, Chiba University, Chiba, Chiba, Japan

${ }^{19}$ Department of Physics, School of Natural Sciences, Ulsan National Institute of Science and Technology, UNIST-gil, Ulsan, Korea

${ }^{20}$ Institute of Particle and Nuclear Studies, KEK, Tsukuba, Ibaraki, Japan

${ }^{21}$ Faculty of Science, Kochi University, Kochi, Kochi, Japan

${ }^{22}$ Department of Physical Sciences, Ritsumeikan University, Kusatsu, Shiga, Japan

${ }^{23}$ Department of Physics, Sungkyunkwan University, Jang-an-gu, Suwon, Korea

${ }^{24}$ Service de Physique Théorique, Université Libre de Bruxelles, Brussels, Belgium

25 Department of Physics and Astronomy, Rutgers University, The State University of New Jersey, Piscataway, NJ, USA

${ }^{26}$ Earthquake Research Institute, University of Tokyo, Bunkyo-ku, Tokyo, Japan

${ }^{27}$ Graduate School of Information Sciences, Hiroshima City University, Hiroshima, Hiroshima, Japan

${ }^{28}$ Advanced Science Institute, RIKEN, Wako, Saitama, Japan

${ }^{29}$ National Institute of Radiological Science, Chiba, Chiba, Japan

${ }^{30}$ Department of Physics, Ehime University, Matsuyama, Ehime, Japan

Received 2014 April 25; accepted 2014 June 26; published 2014 July 14

\begin{abstract}
We have searched for intermediate-scale anisotropy in the arrival directions of ultrahigh-energy cosmic rays with energies above $57 \mathrm{EeV}$ in the northern sky using data collected over a $5 \mathrm{yr}$ period by the surface detector of the Telescope Array experiment. We report on a cluster of events that we call the hotspot, found by oversampling using $20^{\circ}$ radius circles. The hotspot has a Li-Ma statistical significance of 5.1 $\sigma$, and is centered at R.A. $=146.7$, decl. $=43^{\circ} .2$. The position of the hotspot is about $19^{\circ}$ off of the supergalactic plane. The probability of a cluster of events of $5.1 \sigma$ significance, appearing by chance in an isotropic cosmic-ray sky, is estimated to be $3.7 \times$ $10^{-4}(3.4 \sigma)$.
\end{abstract}

Key words: acceleration of particles - cosmic rays - large-scale structure of universe - surveys

Online-only material: color figure, machine-readable table 


\section{INTRODUCTION}

The origin of ultrahigh-energy cosmic rays (UHECRs), particles with energies greater than $10^{18} \mathrm{eV}$, is one of the mysteries of astroparticle physics. Greisen, Zatsepin, and Kuz'min (GZK) predicted that UHECR protons with energies greater than $\sim 60 \mathrm{EeV}\left(6 \times 10^{19} \mathrm{eV}\right)$ would be severely attenuated primarily due to pion photoproduction interactions with the cosmic microwave background radiation (Greisen 1966; Zatsepin \& Kuz'min 1966). This GZK suppression becomes strong if these very high energy cosmic rays are produced at and traveling moderate extragalactic distances. The High Resolution Fly's Eye (HiRes) collaboration was first to observe a suppression of cosmic rays above $\sim 60 \mathrm{EeV}$ Abbasi et al. (2008), which is consistent with expectation from the GZK cutoff. This suppression was independently confirmed by both the Pierre Auger Observatory (PAO; Abraham et al. 2008) in the south and Telescope Array (TA) experiment (Abu-Zayyad et al. 2013a) in the north, which are the largest aperture cosmic-ray detectors currently in operation.

The distribution of UHECR sources should be limited within the local universe with distances smaller than $100 \mathrm{Mpc}$ for proton/iron and $20 \mathrm{Mpc}$ for helium/carbon/nitrogen/oxygen (distances within which $\sim 50 \%$ of cosmic rays are estimated to survive; Kotera \& Olinto 2011). To accelerate particles up to the ultrahigh-energy region, particles must be confined to the accelerator site for more than a million years by a magnetic field and/or a large-scale confinement volume (Hillas 1984; Ptitsyna $\&$ Troitsky 2010). This would thus limit the number of possible accelerators in the universe to astrophysical candidates such as galaxy clusters, supermassive black holes in active galactic nuclei (AGNs), jets and lobes of active galaxies, starburst galaxies, gamma-ray bursts, and magnetars. Galactic objects are not likely to be the sources since past observations indicate that the UHECRs do not concentrate in the galactic plane and have a relatively isotropic distribution. In addition, our Galaxy cannot confine UHECRs above $10^{19} \mathrm{eV}$ within its volume by the GMF. Extragalactic astrophysical objects form well-known large-scale structures (LSSs), most of which are spread along the "supergalactic plane" in the local universe. Nearby AGNs are clustered and concentrated around LSS with a typical clustering length of 5-15 Mpc, as observed by Swift BAT (Cappelluti et al. 2010). Concentrations of nearby AGNs coincide spatially with the LSS of matter in the local universe, including galaxy clusters such as Centaurus and Virgo. The typical amplitude of such AGN concentrations is estimated to be a few hundred percent of the averaged density within a $20^{\circ}$ radius circle, which is of an angular scale comparable to the clustering length of the AGNs within $85 \mathrm{Mpc}$ (Ajello et al. 2012).

The main difficulty in identifying the origin of UHECRs is the loss of directional information due to magnetic field induced bending. In order to investigate the UHECR propagation from the extragalactic sources, a number of numerical simulations have been developed (Yoshiguchi et al. 2003; Sigl et al. 2004; Takami et al. 2006; Kashti \& Waxman 2008; Koers \& Tinyakov 2009; Takami \& Sato 2010; Kalli et al. 2011; Takami et al. 2012). In the simulations, the UHECR trajectory between the assumed UHECR source and the Earth is traced through intergalactic and galactic magnetic fields (IGMF and GMF). The results depend strongly on the assumed distribution and density of the UHECR sources and the intervening magnetic fields. The deflection angle of a $60 \mathrm{EeV}$ proton from a source at a distance of $50 \mathrm{Mpc}$ is estimated to be a few degrees assuming models with an IGMF strength of $1 \mathrm{nG}$. Meanwhile, the estimated deflection by the GMF ranges from a few to about $10^{\circ}$. This, however, depends on the direction in the sky. If the highest-energy cosmic rays come from the local universe such as nearby galaxies, and if they are protons, the maximum amplitude of the cosmic-ray anisotropy above $\sim 60 \mathrm{EeV}$ is expected to be a few hundred percent of the average cosmic-ray flux. In this case, the amplitude of the cosmic-ray anisotropy might be detectable by the UHECR detectors of the TA and PAO.

In the highest-energy region, $E>57 \mathrm{EeV}$, the PAO found correlations of the cosmic-ray directions within a 3.1 radius circle centered at nearby AGNs (within $75 \mathrm{Mpc}$ ) in the southern sky (Abraham et al. 2007). Updated measurements from the PAO indicate a weakened correlation with nearby AGNs (Abreu et al. 2010; Macolino 2012); the correlating fraction (the number of correlated events divided by all events) decreased from the early estimate of $\left(69_{-13}^{+11}\right) \%$ to $(33 \pm 5) \%$, compared with $21 \%$ expected for an isotropic distribution of cosmic rays. The chance probability of the original $(69 \%)$ correlation is $6 \times 10^{-3}$ assuming an isotropic sky. The TA has also searched for UHECR anisotropies such as autocorrelations, correlations with AGNs, and correlations with the LSS of the universe using the first 40 months of scintillator surface detector (SD) data (Abu-Zayyad et al. 2012b, 2013b). Using $5 \mathrm{yr}$ of SD data, we updated results of the cosmic-ray anisotropy with $E>57 \mathrm{EeV}$, which shows deviations from isotropy at the significance of $2 \sigma-3 \sigma$ (Fukushima et al. 2013). In this Letter, we report on indications of intermediate-scale anisotropy of cosmic rays with $E>57 \mathrm{EeV}$ in the northern hemisphere sky using the $5 \mathrm{yr} \mathrm{TA}$ SD data set.

\section{EXPERIMENT}

The TA is the largest cosmic-ray detector in the northern hemisphere. It consists of a scintillator SD array (Abu-Zayyad et al. 2012a) and three fluorescence detector (FD) stations (Tokuno et al. 2012). The observatory has been in full operation in Millard Country, Utah, USA (39.30N, 112.91W; about $1400 \mathrm{~m}$ above sea level) since 2008. The TA SD array consists of 507 plastic scintillation detectors each $3 \mathrm{~m}^{2}$ in area and located on a $1.2 \mathrm{~km}$ square grid. The array has an area of $\sim 700 \mathrm{~km}^{2}$. The TA SD array observes cosmic-ray-induced extensive air showers with $E>\sim 1 \mathrm{EeV}$, regardless of weather conditions with a duty cycle near $100 \%$ and a wide field of view (FoV). These capabilities ensure a very stable and large geometrical exposure over the northern sky survey in comparison with FD observations that have a duty cycle of $\sim 10 \%$.

\section{DATA SET}

In this analysis, we used SD data recorded between 2008 May 11 and 2013 May 4. The dataset contains approximately 1 million triggered events. For the reconstructed events, the energies determined by the SD array were renormalized by $1 / 1.27$ to match the SD energy scale to that of the FD, which was determined calorimetrically (Abu-Zayyad et al. 2013a). Of these events, 72 met the following conditions: (1) each event included at least four SD counters, (2) the zenith angle of the event arrival direction was less than $55^{\circ}$, and (3) the reconstructed energy was greater than $57 \mathrm{EeV}$, which corresponds to the energy threshold determined from the AGN correlation analysis results obtained by the PAO (Abraham et al. 2007), and is adopted here to avoid introducing a free parameter in the scanning phase space. 
(a)

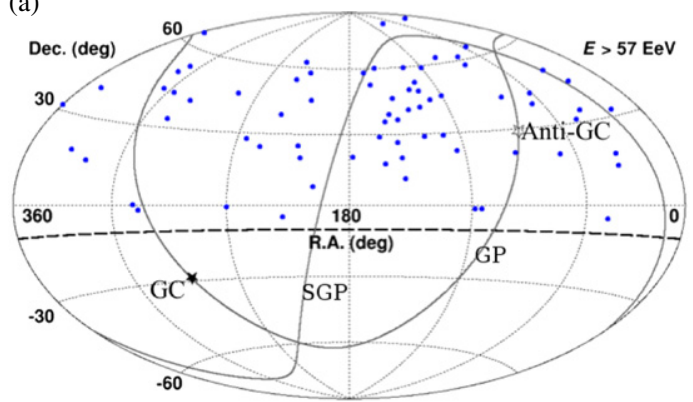

(c)

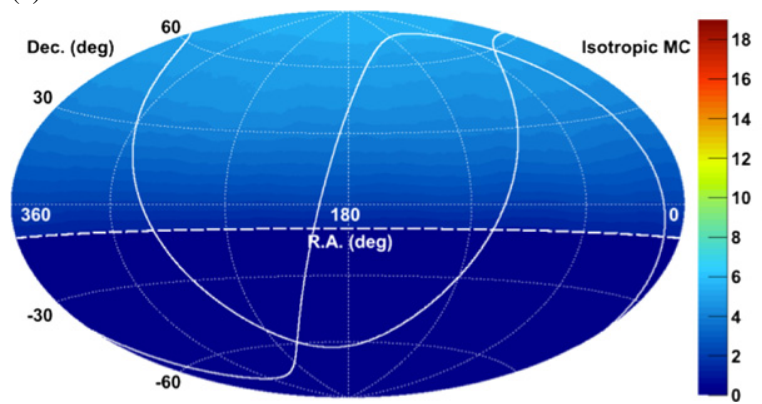

(b)

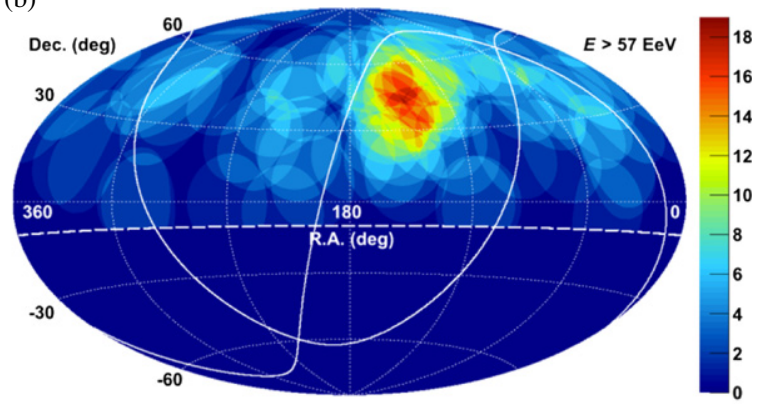

(d)

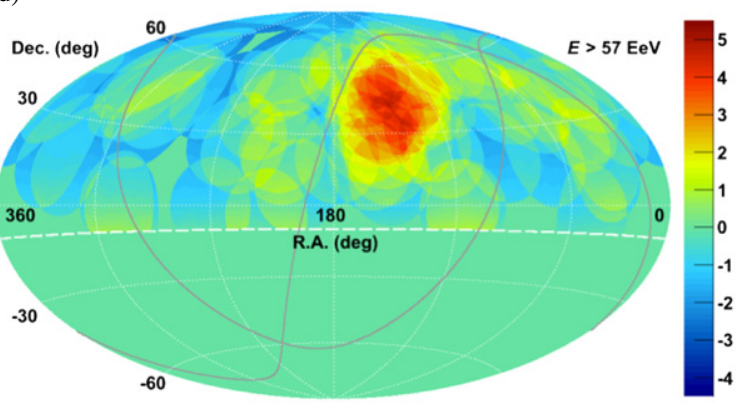

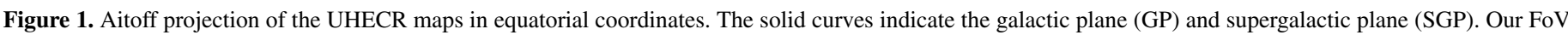

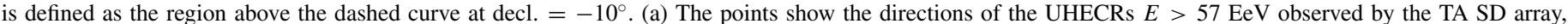

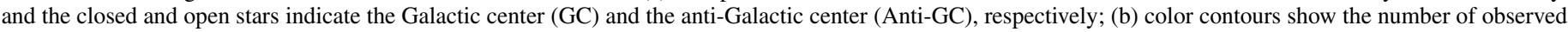

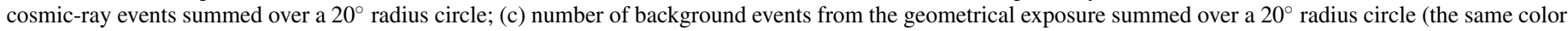
scale as (b) is used for comparison); (d) significance map calculated from (b) and (c) using Equation (1).

The event selection criteria above are somewhat looser than those of our previous analyses of cosmic-ray anisotropy (Fukushima et al. 2013) to increase the observed cosmic-ray statistics. In our previous analyses, the largest signal counter is surrounded by four working counters that are its nearest neighbors to maintain the quality of the energy resolution and angular resolution. Only 52 events survived those tighter cuts. When the edge cut is abolished from the analysis (presented here) to keep more cosmic-ray events, 20 events with $E>$ $57 \mathrm{EeV}$ are recovered compared with the tighter cut analysis. A full Monte Carlo (MC) simulation, which includes detailed detector responses (Abu-Zayyad et al. 2013a), predicted a 13.2 event increase in the number of events. The chance probability of the data increment being 20 as compared to the MC prediction of 13.2 is estimated to be $5 \%$, which is within the range of statistical fluctuations. The angular resolution of array boundary events deteriorates to 1.7 , compared to 1.0 for the well contained events. The energy resolution of array boundary events also deteriorates to $\sim 20 \%$, where that of the inner array events is $\sim 15 \%$. These resolutions are still good enough to search for intermediate-scale cosmic-ray anisotropy. One final check is that when we calculate the cosmic-ray spectrum using the loose cuts analysis, the result is consistent with our published spectrum.

\section{RESULTS}

Figure 1(a) shows a sky map in equatorial coordinates of the 72 cosmic-ray events with energy $E>57 \mathrm{EeV}$ observed by the TA SD array. A cluster of events appears in this map centered near right ascension $\sim 150^{\circ}$, and declination $\sim 40^{\circ}$, with a diameter of $\sim 30^{\circ}-40^{\circ}$. In order to determine the characteristics of the cluster, and estimate the significance of this effect, we choose to apply elements of an analysis that was developed by the AGASA collaboration to search for large- size anisotropy (Hayashida et al. 1999a, 1999b), namely to use oversampling with a $20^{\circ}$ radius. Being mindful that scanning the parameter space of the analysis causes a large increase in chance corrections, we have not varied this radius. The TA and HiRes collaborations used this method previously (Kawata et al. 2013; Ivanov et al. 2007) to test the AGASA intermediatescale anisotropy results with their data in the $10^{18} \mathrm{eV}$ range. The present letter reports on an extension of this method with application to the $E>57 \mathrm{EeV}$ energy region.

In our analysis, at each point in the sky map, cosmicray events are summed over a $20^{\circ}$ radius circle as shown in Figure 1(b). The centers of tested directions are on a $0.1 \times 0.1$ grid from $0^{\circ}$ to $360^{\circ}$ in right ascension (R.A.) and $-10^{\circ}-90^{\circ}$ in declination (decl.). We found that the maximum of $N_{\text {on }}$, the number of observed events in a circle of $20^{\circ}$ radius is 19 within the TA FoV. To estimate the number of background events under the signal in $N_{\text {on }}$, we generated 100,000 events assuming an isotropic flux. We used a geometrical exposure $g(\theta)=\sin \theta \cos \theta$ as a function of zenith angle $(\theta)$ because the detection efficiency above $57 \mathrm{EeV}$ is $\sim 100 \%$. The zenith angle distribution deduced from the geometrical exposure is consistent with that found in a full MC simulation. The MC generated events are summed over each $20^{\circ}$ radius circle in the same manner as the data analysis, and the number of events in each circle is defined as $N_{\text {off }}$. Figure 1(c) shows the number of background events $N_{\mathrm{bg}}=\eta N_{\text {off }}$, where $\eta=72 / 100,000$ is the normalization factor.

We calculated the statistical significance of the excess of events compared to the background events at each grid point of sky using the following equation (Li \& Ma 1983):

$S_{\mathrm{LM}}=\sqrt{2}\left[N_{\text {on }} \ln \left(\frac{(1+\eta) N_{\text {on }}}{\eta\left(N_{\text {on }}+N_{\text {off }}\right)}\right)+N_{\text {off }} \ln \left(\frac{(1+\eta) N_{\text {off }}}{N_{\text {on }}+N_{\text {off }}}\right)\right]^{1 / 2}$. 


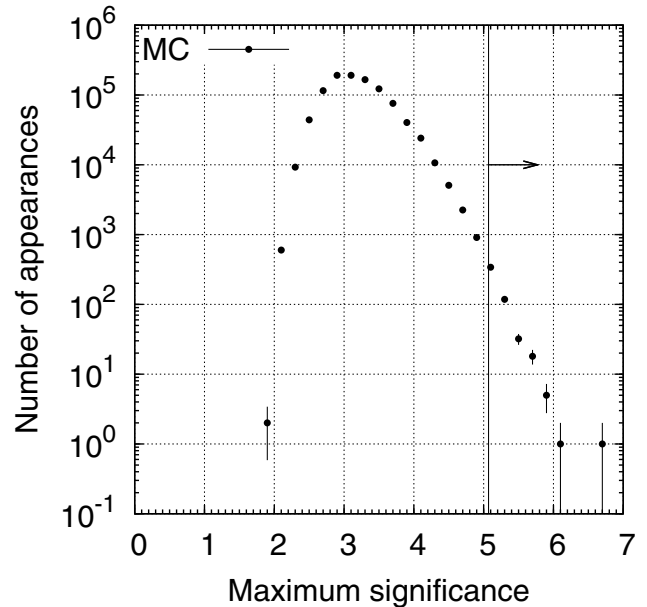

Figure 2. Distribution of the maximum significance in our FoV as determined by a simple MC simulation assuming an isotropic flux. In the set of 1 million trials, each with 72 events, there were 365 instances of $S_{\text {MAX }}>5.1 \sigma$. This is indicated by the solid line and arrow in the plot.

Figure 1(d) shows a significance map (in equatorial coordinates) of the events above $57 \mathrm{EeV}$ as observed by the TA SD array. The maximum excess in our FoV appears as a "hotspot" centered at R.A. $(\alpha)=146.7, \operatorname{decl} .(\delta)=43.2$ with a statistical significance of $S_{\mathrm{MAX}}=5.1 \sigma\left(N_{\mathrm{on}}=19, N_{\mathrm{bg}}=4.49\right)$.

The significance of the hotspot, quoted above at $5.1 \sigma$, does not take random clustering into account, so one must make a correction. We did not carry out a blind analysis, but have been watching the hotspot grow over several years as we collected further data and added events to the sky plot. It is difficult to estimate the penalty due to our having seen the cluster of events. For example, in applying the oversampling technique used by the AGASA experiment, we knew the oversampling radius roughly matched the size of the hotspot cluster.

However, by making a simple MC calculation, one can estimate the probability of such a hotspot appearing by chance anywhere in an isotropic sky. One generates many isotropic MC event sets, each with the statistics of the experimental data, then performs a calculation of the Li-Ma significance exactly as was done on the data; i.e., using oversampling with a radius of $20^{\circ}$. One can go further and approximate the effect of the eye's estimate of the radius of the cluster of events by repeating the calculation at other oversampling radii. We did this, choosing five oversampling radii, $15^{\circ}, 20^{\circ}, 25^{\circ}, 30^{\circ}$, and $35^{\circ}$. We chose a $5^{\circ}$ scan since by eye one cannot make an estimate more accurately than about $\pm 5^{\circ}$.

We generated 1 million MC data sets, each having 72 spatially random events within our FOV (i.e., we reproduced the statistics of the experimental data), assuming a uniform distribution over the TA SD exposure. The maximum of the significances, $S_{\mathrm{MAX}}$, was calculated for each MC data set in the same way as in the data, with the exception that the five oversampling radii were used, and the largest $S_{\text {MAX }}$ was chosen. The distribution of the largest $S_{\text {MAX }}$ of the 1 million data sets is shown in Figure 2. We found that there were 365 instances of $S_{\mathrm{MAX}}>5.1 \sigma$. This yields a chance probability of the observed hotspot in an isotropic cosmic-ray sky of $3.7 \times 10^{-4}$, equivalent to a one-sided probability of $3.4 \sigma$.

To estimate the size of the hotspot, we present (see Figure 3) the normalized number of events as a function of the opening angle, $\psi$, relative to the center of the hotspot in the data. Although with current statistics we cannot determine the shape

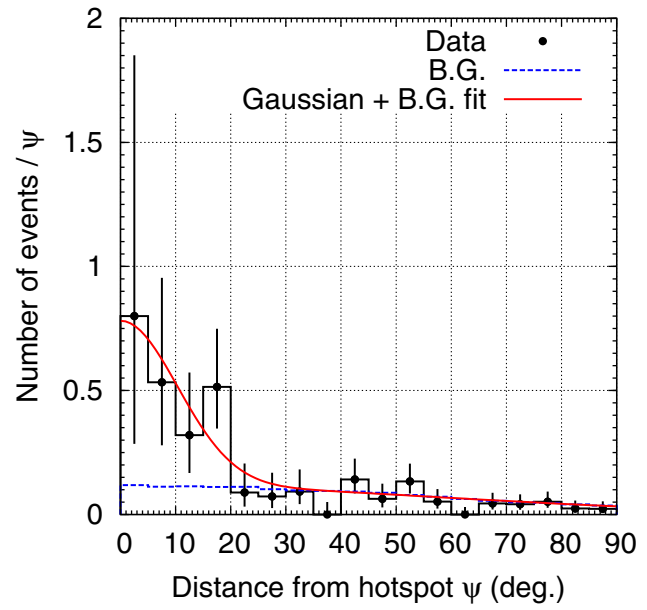

Figure 3. Normalized number of events as a function of the opening angle $(\psi)$ relative to the hotspot. The histogram shown in black with the points shows the observed events above $57 \mathrm{EeV}$ by the TA SD. The dashed blue histogram shows the background events calculated by the MC simulation. The solid red curve is a fit using the binned maximum likelihood method and a Gaussian function with the background calculated with Equation (2). The signal spread and height are estimated to be $\sigma_{\mathrm{s}}=10.3 \pm 1.9$ and $A_{\mathrm{s}}=0.67 \pm 0.29$, respectively, assuming a Gaussian shape.

(A color version of this figure is available in the online journal.)

of the hotspot, to estimate its overall size we fit the hotspot excess using the binned maximum likelihood method, assuming a Gaussian signal plus a background estimated by the MC simulation. We used the following equation:

$$
f\left(\psi ; A_{\mathrm{s}}, \sigma_{\mathrm{s}}\right)=A_{\mathrm{s}} \exp \left(-\frac{\psi^{2}}{2 \sigma_{\mathrm{s}}^{2}}\right)+\left(a_{0}+a_{1} \psi^{2}+a_{2} \psi^{4}\right)
$$

where the first term is the Gaussian signal, and $A_{\mathrm{s}}$ and $\sigma_{\mathrm{s}}$ denote fitting parameters of the signal height and spread, respectively. The second term is the shape of the background fitted by a polynomial function determined from the MC simulations $\left(a_{0}=0.118, a_{1}=-1.7 \times 10^{-5}\right.$, and $\left.a_{2}=8.5 \times 10^{-10}\right)$. The spread of the hotspot was $\sigma_{\mathrm{s}}=10.3 \pm 1.9\left(A_{\mathrm{s}}=0.67 \pm 0.29\right)$. The uncertainty in the position of the hotspot is estimated to be $\sigma_{\mathrm{s}} / \sqrt{N_{\text {on }}-N_{\text {bg }}}=2.7$.

\section{DISCUSSION}

There are no known specific sources behind the hotspot. The hotspot is located near the supergalactic plane, which contains local galaxy clusters such as the Ursa Major cluster $(20 \mathrm{Mpc}$ from Earth), the Coma cluster (90 Mpc), and the Virgo cluster $(20 \mathrm{Mpc})$. The angular distance between the hotspot center and the supergalactic plane in the vicinity of the Ursa Major cluster is $\sim 19^{\circ}$.

Assuming the hotspot is real, two possible interpretations are that it may be associated with the closest galaxy groups and/ or the galaxy filament connecting us with the Virgo cluster (Dolag et al. 2004); or, if cosmic rays are heavy nuclei, they may originate close to the supergalactic plane and be deflected by extragalactic magnetic fields and the galactic halo field (Tinyakov \& Tkachev 2002; Takami et al. 2012). To determine the origin of the hotspot, we will need greater UHECR statistics in the northern sky. Better information about the mass composition of the UHECRs, GMF, and IGMF would also be important. 


\section{SUMMARY}

Using cosmic-ray events with energy $E>57 \mathrm{EeV}$, collected over five years with the TA SD, we have observed a cluster of events, which we call the hotspot, with a statistical significance of $5.1 \sigma\left(N_{\text {on }}=19, N_{\mathrm{bg}}=4.49\right)$, centered at R.A. $=146^{\circ} .7$, decl. $=43.2$. We calculated the probability of such a hotspot appearing by chance in an isotropic cosmic-ray sky to be $3.7 \times$ $10^{-4}(3.4 \sigma)$.

This indication of intermediate-scale anisotropy is limited by statistics collected by experiments in the northern hemisphere. It provides a strong impetus for an improved effort to study the origin of UHECRs. The TA $\times 4$ project (extension of the TA SD by a factor of 4; Sagawa 2013) is designed to provide the equivalent of 20 TA-years of SD data by 2019, which would yield a $\sim 7 \sigma$ observation if the ratio of hotspot to background events remains as is currently seen. TA $\times 4$ and other related projects will enable us to make a precise UHECR anisotropy map with high statistics and help solve the mystery of the UHECR origin.

The Telescope Array experiment is supported by the Japan Society for the Promotion of Science through Grants-inAids for Scientific Research on Specially Promoted Research (21000002) "Extreme Phenomena in the Universe Explored by Highest Energy Cosmic Rays" and for Scientific Research (19104006), and the Inter-University Research Program of the Institute for Cosmic Ray Research; by the U.S. National Science Foundation awards PHY-0307098, PHY0601915, PHY-0649681, PHY-0703893, PHY-0758342, PHY0848320, PHY-1069280, and PHY-1069286; by the National Research Foundation of Korea (2007-0093860, R32-10130, 2012R1A1A2008381, 2013004883); by the Russian Academy of Sciences, RFBR grants 11-02-01528a and 13-02-01311a (INR), IISN project No. 4.4509.10 and Belgian Science Policy under IUAP VII/37 (ULB). The foundations of Dr. Ezekiel R. and Edna Wattis Dumke, Willard L. Eccles and the George S. and Dolores Dore Eccles all helped with generous donations. The State of Utah supported the project through its Economic Development Board, and the University of Utah through the Office of the Vice President for Research. The experimental site became available through the cooperation of the Utah School and Institutional Trust Lands Administration (SITLA), U.S. Bureau of Land Management, and the U.S. Air Force. We also wish to thank the people and the officials of Millard County, Utah for their steadfast and warm support. We gratefully acknowledge the contributions from the technical staffs of our home institutions. An allocation of computer time from the Center for High Performance Computing at the University of Utah is gratefully acknowledged.

\section{APPENDIX}

\section{LIST OF EVENTS WITH $E>57 \mathrm{EeV}$}

In this Appendix we present the list of events with energy $E>57 \mathrm{EeV}$ and zenith angle $\theta<55^{\circ}$ that have been recorded by the SD of the TA from 2008 May 11 to 2013 May 4. During this period, 72 such events were observed. Table 1 shows the arrival date and time of these events, the zenith angle, energy in units of $\mathrm{EeV}$, and equatorial coordinates $\alpha$ (R.A.) and $\delta$ (decl.) in degrees. Noted that the air shower reconstruction used here as
Table 1

List of Telescope Array Events with $E>57 \mathrm{EeV}$ and Zenith Angle $\theta<55^{\circ}$ Recorded from 2008 May 11 to 2013 May 4

\begin{tabular}{lcrrr}
\hline \hline $\begin{array}{l}\text { Date and Time } \\
\text { (UTC) }\end{array}$ & $\begin{array}{c}\theta \\
(\mathrm{deg})\end{array}$ & $\begin{array}{c}E \\
(\mathrm{EeV})\end{array}$ & $\begin{array}{c}\alpha \\
(\mathrm{deg})\end{array}$ & $\begin{array}{c}\delta \\
(\mathrm{deg})\end{array}$ \\
\hline 2008 Jun 10 17:05:37 & 46.91 & 88.8 & 93.50 & 20.82 \\
2008 Jun 25 19:45:52 & 31.98 & 82.6 & 68.86 & 19.20 \\
2008 Jun 29 08:22:45 & 41.20 & 101.4 & 285.74 & -1.69 \\
2008 Jul 15 05:26:31 & 34.26 & 57.3 & 308.45 & 53.91 \\
2008 Jul 20 04:35:32 & 25.61 & 120.3 & 285.46 & 33.62 \\
2008 Aug 1 23:01:33 & 39.43 & 139.0 & 152.27 & 11.10 \\
2008 Aug 9 06:16:16 & 14.54 & 76.9 & 280.28 & 41.34 \\
2008 Aug 10 12:45:04 & 38.04 & 122.2 & 347.73 & 39.46 \\
2008 Sep 24 20:09:22 & 23.16 & 68.8 & 178.03 & 20.29 \\
2008 Oct 8 18:20:16 & 24.69 & 69.1 & 154.49 & 26.50 \\
\hline
\end{tabular}

(This table is available in its entirety in a machine-readable form in the online journal. A portion is shown here for guidance regarding its form and content.)

described in Kawata et al. (2013) was slightly different from that of previous anisotropy work (Abu-Zayyad et al. 2012b). The opening angles between these arrival directions and previous ones are almost within $1^{\circ}$. This difference hardly affects the results of the large-scale anisotropy.

\section{REFERENCES}

Abbasi, R. U., Abu-Zayyad, T., Allen, M., et al. 2008, PhRvL, 100, 101101 Abraham, J., Abreu, P., Aglietta, M., et al. 2007, Sci, 318, 938 Abraham, J., Abreu, P., Aglietta, M., et al. 2008, PhRvL, 101, 061101 Abreu, P., Aglietta, M., Ahn, E. J., et al. 2010, APh, 34, 314 Abu-Zayyad, T., Aida, R., Allen, M., et al. 2012a, NIM-A, 689, 87 Abu-Zayyad, T., Aida, R., Allen, M., et al. 2012b, ApJ, 757, 26 Abu-Zayyad, T., Aida, R., Allen, M., et al. 2013a, ApJL, 768, L1 Abu-Zayyad, T., Aida, R., Allen, M., et al. 2013b, ApJ, 777, 88 Ajello, M., Alexander, D. M., Greiner, J., et al. 2012, ApJ, 749, 21 Cappelluti, M., Ajello, M., Burlon, D., et al. 2010, ApJL, 716, L209 Dolag, K., Grasso, D., Springel, V., \& Tkachev, I. 2004, JKAS, 37, 427

Fukushima, M., Ivanov, D., Kawata, K., et al. 2013, in Proc. of 33rd ICRC (Rio de Janeiro), CR-EX, Id:1033

Greisen, K. 1966, PhRvL, 16, 748

Hayashida, N., Nagano, M., Nishikawa, D., et al. 1999a, APh, 10, 303

Hayashida, N., Honda, K., Inoue, N., et al. 1999b, in Proc. of 26th ICRC (Salt Like CIty), ed. D. Kieda, M. Salamon, \& B. Dingus (Melville, NY: AIP), 3, 256

Hillas, A. M. 1984, ARA\&A, 22, 425

Ivanov, D., \& Thomson, G.B. The HiRes Collaboration 2007, in Proc. of 30th ICRC (Merida), ed. R. Caballero et al. (Mexico City, Mexico: Universidad Nacional Autonoma de Mexico), 4, 445

Kalli, S., Lemoine, M., \& Kotera, K. 2011, A\&A, 528, 109

Kashti, T., \& Waxman, E. 2008, JCAP, 005, 006

Kawata, K., Fukushima, M., Ikeda, D., et al. 2013, in Proc. of 33rd ICRC (Rio de Janeiro), CR-EX, Id:311

Koers, H. B. J., \& Tinyakov, P. 2009, JCAP, 0904, 003

Kotera, K., \& Olinto, A. V. 2011, ARA\&A, 49, 119

Li, T.-P., \& Ma, Y.-Q. 1983, ApJ, 272, 317

Macolino, C. 2012, JPhCS, 375, 052002

Ptitsyna, K. V., \& Troitsky, S. V. 2010, PhyU, 53, 691

Sagawa, H. 2013, in Proc. of 33rd ICRC (Rio de janeiro), CR-EX, Id:121

Sigl, G., Miniati, F., \& Ensslin, T. A. 2004, PhRvD, 70, 043007

Takami, H., Inoue, S., \& Yamamoto, T. 2012, APh, 35, 767

Takami, H., \& Sato, K. 2010, ApJ, 724, 1456

Takami, H., Yoshiguchi, H., \& Sato, K. 2006, ApJ, 639, 803

Tinyakov, P. G., \& Tkachev, I. I. 2002, APh, 18, 165

Tokuno, H., Tameda, Y., Takeda, M., et al. 2012, NIM-A, 676, 54

Yoshiguchi, H., Nagataki, S., Tsubaki, S., \& Sato, K. 2003, ApJ, 586, 1211

Zatsepin, G. T., \& Kuz'min, V. A. 1966, JETPL, 4, 78 